\section{Estimation of Heterogeneous Error Variances}

We consider a set of $p q$ observations $x_{i j}$ in a twoway classification, which we may suppose represented by the model

$x_{i j}=\alpha_{i}+\beta_{j}+\varepsilon_{i j}(i=1,2, \ldots p ; j=1,2, \ldots q)$, where $\alpha_{i}$ and $\beta_{j}$ are constants and the $\varepsilon_{i j}$ are independent normal random errors with zero means and variances $r_{j}^{2} ; \alpha_{i}, \beta_{j}$ and $c_{j}^{2}$ are unknown, and it is desired to estimate $\sigma_{j}{ }^{2}$. The problem arises, for example, in investigating the accuracy of a foodtasting panel; $\alpha_{i}$ depends on the food-specimen tasted, $\rho_{j}$ is the bias and $\sigma_{j}{ }^{2}$ the residual error variance of the $j$ th panel member.

If $p$ or $q$ is small, the method of maximum likelihood does not give a satisfactory estimate of $\sigma_{j}{ }^{2}$. Kendall ${ }^{1}$ notes that when $q=2$ the maximum likelihood estimates of $\sigma_{1}{ }^{2}$ and $\sigma_{2}{ }^{2}$ are equal, and therefore are not consistent if, in fact, $\sigma_{1}{ }^{2}$ and $\sigma_{2}{ }^{2}$ are unequal.

It is of interest to consider estimates which are, in the first place, unbiased. One such estimate, valid for $q \geqslant 3$, is

$$
\begin{aligned}
s_{j}^{2}= & \frac{q}{(p-1)(q-2)}\left\{\sum_{i}^{p}\left(x_{i j}-x_{i .}-x_{. j}+x_{. .}\right)^{2}-\right. \\
& \left.\frac{1}{q(q-1)} \sum_{i}^{p} \sum_{r}^{q}\left(x_{i r}-x_{i .}-x_{. r}+x_{. .}\right)^{2}\right\},
\end{aligned}
$$

where $x_{i}$. and $x_{. j}$ denote partial means and $x_{\text {. }}$ the overall mean of $x_{i j}$. Another estimate, valid for $q \geqslant 2$, is

$$
\begin{aligned}
s_{j}{ }^{2}=\frac{1}{p-1}\left\{\sum_{i}\left(x_{i j}-x_{\cdot j}\right)^{2}-\frac{2}{q(q-1)}\right. & \left.\sum_{i} \sum_{r>8}\left(x_{i r}-x_{. r}\right)\left(x_{i s}-\dot{x}_{\cdot s}\right)\right\} .
\end{aligned}
$$

When $q=2$ this gives, for $j=1$, say,

$$
s_{1}^{\prime 2}=\frac{1}{p-1} \sum_{i}\left(x_{i_{1}}-x_{\cdot_{1}}\right)\left(x_{i_{1}}-x_{\bullet_{1}}-x_{i_{2}}+x_{\bullet_{2}}\right),
$$

which can be shown to be the only unbiased quadratic-form estimate of $\sigma_{1}{ }^{2}$.

The variances of these estimates of $c_{j}^{2}$ depend on other unknowns besides $c_{j}^{2}$ itself. We find

$$
\begin{aligned}
& \operatorname{var}\left(s_{j}{ }^{2}\right)=\frac{2 \sigma^{4}}{p-1}+\frac{4}{(p-1)(q-1)^{2}} \\
&\left\{\epsilon_{j}^{2} \sum_{r \neq j}^{\sum c_{r}+\frac{1}{(q-2)^{2}}} \sum_{\substack{r>8 \\
r, s \neq j}} \sigma_{r}^{2} \sigma_{s}^{2}\right\},
\end{aligned}
$$

which depends on all of $\sigma_{1}^{2}, c_{2}^{2}, \ldots . \sigma_{q}^{2}$; and

$$
\begin{aligned}
& \operatorname{var}\left(s_{j}{ }^{2}\right)= \frac{2 \sigma j^{4}}{p-1}+\frac{p-2}{p(p-1)^{2} q^{2}} \sum_{i}\left(\alpha_{i}-\alpha_{.}\right)^{2} \\
&\left\{(q-1)^{2} \sigma_{j}^{2}+\sum_{r \neq j} \sigma_{r^{2}}\right\}+ \\
& \frac{4}{(p-1) q^{2}(q-1)^{2}} \underset{r>8}{\sum \sigma_{r} \sigma_{s}^{2},}
\end{aligned}
$$

which depends in addition on $\alpha_{1}, \alpha_{2}, \ldots \alpha_{p}$. When $q>2$ and both estimates are available, sometimes one and sometimes the other has the lower variance, according to the values of the other parameters.
A full discussion is in preparation. The investigation is in connexion with work done on behalf of the Department of Scientific and Industrial Research (Food Investigation).

Statistical Laboratory,

University of Cambridge. April 19.

'Kendall, M. G., J. Roy. Statist. Soc., B (In the press).

\section{A. S. C. EhrEnBERg}

\section{Changes in the Histology of the Honey-bee Ventriculus Associated with the Ingestion of Certain Insecticides}

Although the histological changes produced by insecticides have been the object of study by a few investigators $^{1}$, the subject has on the whole received little attention. Recently a comparative study of the histology of the honey-bee ventriculus, following the administration, in individual graded doses, of the insecticides, acid lead arsenate, DDT and 'Parathion', has been made. In addition, the median lethal doses for the insecticides were determined, and observations on the behaviour of the poisoned bees and the macroscopical appearance of the mid.guts (ventriculi) were made. For the microscopical studies material was prepared with only slight modifications of standard histological procedures. (The name 'Parathion' is used here for the compound o,o-diethyl $o, p$-nitrophenyl thiophosphate.)

It was found that in some instances there were definite histological abnormalities following the administration of the insecticides. When present, these abnormalities were mostly confined to the middle region of the ventriculus. Photomicrographs of transverse sections through this region of the midgut are shown. Fig. 1 is that from a normal bee; Fig. 2 from an arsenic-poisoned bee; Fig. 3 from a DDT-poisoned bee ; and Fig. 4 from a 'parathion'poisoned bee.

Observations on the macroscopic appearance of mid-guts from arsenic-poisoned bees showed that all were lighter in colour than those from normal bees, and that 90 per cent contained a greyish plug-like mass near their posterior end. Microscopically, midguts from all arsenic-poisoned bees presented the same appearance: severe vacuolization, desquamation and degeneration of the epithelial cells were characteristic (Fig. 2). Similar effects have been observed in various experiments with other test insects ${ }^{2,3}$.

The mid-guts of 50-60 per cent of the DDT. poisoned bees contained a large transparent gas bubble, and a microscopical study of this region (Fig. 3) showed that the epithelial lining was stretched and that increased proliferation, vacuolization and secretion from the epithelial cells were taking place. The epithelial lining of mid-guts containing no gas bubble was not stretched appreciably; but the condition and activity of the epithelial cells were similar. It is suggested that this activity of the epithelial cells is a result of the hyperactivity of the insect, caused by the action of DD'T on the nervous system.

Both the macroscopic and the microscopic (Fig. 4) appearance of the mid-guts of 'Parathion'-poisoned bees closely resembled those of normal bees. The hyperactivity and characteristic circus movements of a 'Parathion'-poisoned bee indicated that this insecticide was acting as a nerve poison.

A fuller account of this investigation will be given elsewhere. 